\title{
Clinical Profile and Outcome of Poisoned Pediatric Patients Admitted To Poison Control Center, Ain Shams University Hospitals during the Year 2012
}

\author{
Sawsan A. Shalaby, Sonya M. S. Azab, Noha El Rafie ${ }^{1}$ and Hany M.Tawfik² \\ ${ }^{1}$ Forensic Medicine and Clinical Toxicology Department, Faculty of Medicine \\ ${ }^{2}$ Consultant of clinical toxicology and head of the Information System Department, Poison Control Center
}

Ain Shams University, Cairo, Egypt.

\begin{abstract}
Childhood poisoning comprises a significant component of injury-related morbidity and mortality all over the world and exerts a huge amount of pressure on the resources of the health care system as well as the workload on the health care staff. Early and accurate diagnosis and management of poisoning decrease the risk of morbidity and mortality. Therefore, study of manifestations and severity of poisoning by variable agents, effects of treatments and outcome is essential to assess the impact of poisonous substances and the effectiveness of treatment.

Aim of the study: this study aims to determine the clinical profile, management and outcome of acutely poisoned children admitted to Poison Control Center (PCC), Ain Shams University Hospitals during a one year period starting from $1 / 1 / 2012$ to $31 / 12 / 2012$.

Methods: The collected data from the cases' medical records included: age, gender, delay time, pre-consultation treatment, clinical manifestations, findings of investigations, place and period of hospitalization, received treatment in the PCC and the outcome.

Results: adolescence where the most frequent age group in this study and female gender was more prominent than male gender. Delay time was significantly increased in non-survivors than survivors. The proportion of deaths in group of patients who received pre-consultation treatment was significantly higher than that in patients who didn't receive any treatment before arrival to the PCC. Faulty management in the form of induction of emesis by salty water intake was found in $2.6 \%$ of the cases; $7.7 \%$ of these cases were died. Most cases presented with unremarkable changes in vital signs and gastrointestinal manifestations (mainly nausea and vomiting) were the most frequent manifestations in the cases. Hypoglycemia, hyponatremia, hypokalemia and metabolic acidosis were the most common abnormalities found in laboratory investigations. Sinus tachycardia was the most common abnormality found in ECG.

Most of the included cases in this study were admitted in the inpatient section (83\%). The majority of the cases $(75 \%)$ were hospitalized for short period that didn't exceed 24 hours. Higher PSS was associated with longer hospital stay periods. Corrosives, followed by pesticides were the most frequent cause of prolonged hospitalization ( $\geq 4$ days). Emergency managements were indicated in $11 \%$ of the cases and procedures for gastric decontamination were done for 1296 cases $(87.3 \%)$. All cases received supportive and symptomatic treatments. Atropine was the most frequent antidote that was used either alone or with toxogonin.

The overall mortality ratio was $2.2 \%$. Preschool age group had the greatest mortality ratio $(3.7 \%)$, followed by infant group (3.6\%). Hypernatremia had the highest case fatality rate $(100 \%)$, followed by CO poisoning $(28.6 \%)$. Conclusion: Gastrointestinal manifestations (mainly nausea and vomiting) were the most frequent manifestations. Hypoglycemia, hyponatremia, hypokalemia and metabolic acidosis were the most common abnormalities found in laboratory investigations. Sinus tachycardia was the most common abnormality found in ECG. Corrosives, followed by pesticides were the most frequent cause of prolonged hospitalization. Atropine was the most frequently used antidote. The overall mortality was $2.2 \%$. Hypernatremia had the highest case fatality rate (100 $\%)$.

Recommendations: Increasing public awareness of the first aid measures in childhood poisoning is crucial to avoid complications of faulty measures. Also, it is important to increase public awareness of the services of the information center in the PCC as it presents guidance about management of poisoned cases. Ongoing childhood poisoning surveillance is needed to track its effects and risk factors and to monitor the impact of appropriate interventions.
\end{abstract}

Keywords Poisoning, Children, Outcome, Clinical, Management 


\section{Introduction}

$\mathrm{C}$ hildhood poisoning comprises a significant component of injury-related morbidity and Werld According to the WHO world report on child injury prevention, acute poisoning accounts for an estimated 45,000 deaths annually in children and young people under the age of 20 years (Holder et al., 2008).

Acute poisoning in children represents one of the most common medical emergencies encountered in young children, and accounts for a significant proportion of emergency room visits for the adolescent population (Aqeel et al., 2009). It exerts a huge amount of pressure on the resources of the health care system as well as the workload on the health care staff (Randev et al., 2011).

Early and accurate diagnosis and management of poisoning decrease the risk of morbidity and mortality. Therefore, study of manifestations and severity of poisoning by variable agents, effects of treatments and outcome is essential to assess the impact of poisonous substances and effectiveness of treatment.

This study aims to determine the clinical profile, management and outcome of acutely poisoned children admitted to Poison Control Center (PCC), Ain Shams University Hospitals during a one year period starting from 1/1/2012 to $31 / 12 / 2012$.

\section{Subjects and Methods}

This retrospective study was carried out on all acutely poisoned children of both sexes, aged $\leq 18$ years old who were admitted to PCC, Ain Shams University Hospitals, during one year period starting from 1/1/2012 to 31/12/2012.

An official permission was taken from the director of the PCC. The approval of The Local Research Ethics Committee was obtained. All personal data were kept anonymous to ensure confidentiality of records.

The medical records of the pediatric patients were revised and the following data were obtained:

1. Age and gender

2. Delay time in hours.

3. Pre-consultation treatment (at hospital or at home)

4. Clinical manifestations: the routine clinical examination of poisoned cases includes recording of vital signs as well as systematic general examination of all body systems. Cases were categorized as cases with normal vital functions or abnormal according to the normal ranges of vital signs in children (Hutchison et al., 2008 and Schafermayer, 2012).Reed's classification of the level of consciousness (Chadha, 2003) was used for grading of coma in cases with disturbed level of consciousness.
5. Findings of investigations: cases were categorized to normal/abnormal according to the reference ranges.

6. Place and period of hospitalization.

7. Received treatment in the PCC

8. Outcome of the case, either recovery and discharge or death.

9. Severity of poisoning all cases were categorized according to severity of poisoning using poisoning severity score (PSS) (Persson et al., 1998).

\section{Statistical analysis}

of the data was done using Statistical package for Social Science (SPSS) version 19 software. Chi-square test was used for comparison of frequencies of qualitative variables between groups. Spearman correlation test was used to assess the strength of association between grades of PSS (as ordinal categorical variable) and delay time (continuous variable). Non-parametric test (Mann-Whitney test) was used for comparison of delay time between two groups since its distribution was not normal. All measured $(\mathrm{P})$ values were two-sided. $\mathrm{P}<0.05$ was considered significant.

\section{Results}

This study included 1,521 children who were admitted to the PCC and represented about $22 \%$ of the total pediatric cases. For comparison of poisoning between age groups; the included cases were subdivided into: infants (less than one year old), toddlers ( $1:<3$ years old), preschool age group ( $3:<6$ years old), school age group (6: <12 years old) and adolescents (12: 18 years old) (Kail, 2004).

Figure (1) shows the gender distribution in age groups of the included cases. Adolescents represented the most frequent age group among the admitted pediatric cases, followed by toddlers. Female gender was predominant in the adolescent and school age groups while male gender was predominant in younger age groups. This difference was statistically significant.

The delay time in the included cases ranged from 1 to 24 hours. Mann-Whitney test revealed significant increase in the median of delay time in nonsurvivors than that in survivors (table (1)). Spearman correlation test revealed non-significant correlation between delay time and severity of poisoning (table (2)).

There were 63 cases $(4.1 \%)$ who received treatments before arrival to the PCC either at home or at another health care facility (figure (2)). Specific treatment was the most frequent pre-consultation treatment; it included atropine injection (20 cases), toxogonin injection (6 cases) and antivenom ( 2 cases). This was followed by gastric decontamination; gastric lavage (13 cases) and ingestion of activated charcoal (4 cases). 
Induction of emesis by salty water was found as faulty management given to 39 cases, 3 cases of them $(7.7 \%)$ were died.

Figure (3) shows the number of deaths in relation to pre-consultation treatment of the included cases. The proportion of deaths in group of patients who received pre-consultation treatment was significantly higher than that in patients who didn't receive any treatment before arrival to the PCC.

Table (3) shows severity of poisoning according to PSS grading in relation to causative agents of poisoning in the studied cases. Pesticides were the most frequent agent (477 cases, 31.4\%), followed by cardiopulmonary drugs $(383,25.2 \%)$ and drugs acting on CNS $(205,13.5 \%)$.

Regarding the clinical findings of the cases, the majority of the cases were presented in the PCC with unremarkable changes of vital signs. The most common abnormalities were tachycardia (377 cases, $24.8 \%$ ), hypotension ( 45 cases, $2.9 \%$ ), tachypnea (44 cases, $2.8 \%$ ), hypothermia (100 cases, 6.6\%) and disturbed consciousness level with coma grade I (213 cases, 14\%) (table (4)).

Table (5) shows findings of systemic examination. Gastrointestinal manifestations (mainly nausea and vomiting) had the greatest frequency among the included cases (996 cases, 65.5\%), followed by neurological (345 cases, $22.7 \%$ ) and respiratory manifestations (211 cases, $13.9 \%$ ).

Laboratory investigations included investigations for assessment of the patients' general conditions and specific investigations for assessment of the effects of the poisonous agent. Table (6) shows serum levels of electrolytes and blood sugar level. The most common abnormalities were hypokalemia (697 cases, $45.8 \%$ ), followed by hypoglycemia (194 cases, $14.6 \%$ ), hyponatremia (60 cases, $3.9 \%$ ).

Blood gases tests had been done for 330 cases; table (7) shows that, metabolic acidosis was the most common abnormality as it was found in 75 cases (22.7\%), followed by rrespiratory acidosis (45 cases, $13.6 \%)$.

Table (8) shows specific toxicological tests that were done for 487 cases (32\% of all cases). Pseudo-cholinesterase level in blood was the most frequent performed specific test (351 cases, 72.1\%).

Electrocardiographs (ECG) was done for 360 cases $(23.7 \%)$. Sinus tachycardia was the most frequent abnormality (318 cases, $88.4 \%$ ), followed by sinus bradycardia (20 cases, $5.6 \%$ ) (table (9).

Regarding the place of admission in the PCC, most of the cases were admitted in the inpatient section (1262 cases, $83 \%$ ) and 259 cases (17\%) were admitted in the intensive care unit (ICU). Table (10) shows the place of admission in relation to age groups of the included cases. Chi-square statistical analysis showed that, the proportion of admitted cases to ICU was significantly smaller in both adolescents and infants than in other age groups.
Most of the cases were hospitalized for short duration not exceeding 24 hours (1141 cases, 75\%) and $21.5 \%$ (326 cases) were admitted for period ranged from 1:4 days. Only 54 cases $(3.5 \%)$ were hospitalized for a period $\geq 4$ days. Table (11) shows durations of hospital stay in relation to severity of poisoning (according to PSS grades). Severe cases (PSS grades 3 and 4 ) represented the majority of patients who needed hospitalization for long period ( $\geq 4$ days) (48 cases, $88.9 \%)$.

Table (12) shows the poisonous agents responsible for prolonged hospitalization period $(\geq 4$ days). Corrosives, followed by pesticides, were the most common cause of prolonged hospital stay $(29.6 \%$ and $24.1 \%$ respectively). All of the cases were presented with moderate to severe toxicity (PSS grades 2 and 3 ) and $35.2 \%$ (19 cases) of them died.

The received treatment in the PCC included emergency treatments, decontamination procedures, supportive and specific treatments. Emergency treatments were indicated in 172 cases (11.3\%), (figure (4)). The most frequent procedure was endotracheal tube intubation (93 cases, $6.1 \%$ ), followed by oxygen inhalation (74 cases, $4.9 \%$ ) and cardiopulmonary resuscitation ( 5 cases, $0.3 \%$ ).

Mechanical ventilation (MV) was indicated in 63 patients $(4.1 \%)$. Table (13) shows that, pesticides were the most frequent poisonous agent in these cases (16 cases, $25.4 \%$ ), followed by substances of abuse and poisonous gases (10 cases each, 15.9\% each).

Procedures for gastric decontamination were done for 971 cases $(65.4 \%$ of the cases with oral route of exposure). Induction of emesis by syrup of ipecac was the most frequent method, followed by activated charcoal administration (figure (5)).

Regarding supportive treatment, all admitted cases received maintenance intravenous fluids. Table (14) shows that, medications for treatment of gastric irritation (histamine receptor type II $\left(\mathrm{H}_{2}\right)$ blockers, proton pump inhibitors (PPIs)) and anti-emetics were the most frequent supportive treatment given to the admitted cases (856 cases, 56.3\%). Blood transfusion was indicated for two cases; corrosive and snake poisoning.

Specific treatments were indicated in 338 cases (22.2\%). Table (15) shows that, atropine was the most frequent antidote that was used either alone or with toxogonin (202 cases, $59.7 \%$ and 91 cases, $26.9 \%$ respectively).

The outcome of the cases was determined as recovery and discharge from the PCC or death. The percentage of mortality in the admitted cases was $2.2 \%$ (33 cases). Figure (6) shows percentages of mortality in age groups of the included cases. Preschool age group had the greatest percentage $(3.7 \%)$, followed by infants $(3.6 \%)$.

Table (16) shows case fatality rates of poisonous agent in the studied cases. Hypernatremia had the highest case fatality rate $(100 \%)$, followed by CO poisoning (28.6\%). 
Table (1): Mann-Whitney test comparing the delay time between survivor and none survivors of the pediatric poisoned patients admitted to PCC during the year 2012

\begin{tabular}{|c|c|c|}
\hline $\begin{array}{c}\text { Outcome } \\
\text { Delay time }\end{array}$ & $\begin{array}{c}\text { Survivors } \\
(\text { PSS 0,1,2,3) }\end{array}$ & $\begin{array}{c}\begin{array}{c}\text { Non-survivors } \\
\text { (PSS 4) }\end{array} \\
\end{array}$ \\
\hline Number & 1488 & 33 \\
\hline Median & 2 & 3 \\
\hline Minimum & 1 & 1 \\
\hline Maximum & 24 & 14 \\
\hline \multicolumn{3}{|c|}{ P value: $0.014 *$} \\
\hline
\end{tabular}

Table (2): Spearman correlation test between delay time and PSS grades in all pediatric poisoned patients admitted to PCC during the year 2012

\begin{tabular}{|c|l|c|}
\hline Correlation & \multicolumn{2}{|c|}{ PSS } \\
\hline Delay time (in hours) & Number & 1521 \\
\cline { 2 - 3 } & $\mathrm{r}$ & -0.02 \\
\cline { 2 - 3 } & P value & 0.44 \\
\hline
\end{tabular}

$r$ : Correlation coefficient

Table (3): Severity of poisoning according to PSS in relation to causative agents of poisoning in the pediatric poisoned patients admitted to PCC, Ain Shams University Hospitals during the year 2012:

\begin{tabular}{|l|c|c|c|c|c|c|}
\hline \multirow{2}{*}{ Causative agent } & $\mathbf{1}$ & $\mathbf{2}$ & $\mathbf{3}$ & $\mathbf{4}$ & Total \\
\cline { 2 - 7 } & $\mathbf{N}(\boldsymbol{\%})$ & $\mathbf{N}(\boldsymbol{\%})$ & $\mathbf{N}(\boldsymbol{\%})$ & $\mathbf{N}(\boldsymbol{\%})$ & $\mathbf{N}(\boldsymbol{\%})$ & $\mathbf{N}(\boldsymbol{\%})$ \\
\hline Pesticides & $50(33.3)$ & $175(35.4)$ & $204(29.1)$ & $38(26.4)$ & $10(30.3)$ & $477(31.4)$ \\
\hline Cardio-pulmonary drugs & $37(24.7)$ & $143(28.9)$ & $190(27.1)$ & $12(8.3)$ & $1(3)$ & $383(25.2)$ \\
\hline CNS drugs & $10(6.7)$ & $37(7.5)$ & $139(19.9)$ & $12(8.3)$ & $7(21.2)$ & $205(13.5)$ \\
\hline Substances of abuse & $10(6.7)$ & $49(9.9)$ & $52(7.4)$ & $30(20.8)$ & $2(6.1)$ & $143(9.4)$ \\
\hline Chemical substances & $0(0)$ & $17(3.4)$ & $57(8.1)$ & $39(27.1)$ & $6(18.2)$ & $119(7.8)$ \\
\hline Anti-diabetic agents & $27(18)$ & $45(9.1)$ & $7(1)$ & $0(0)$ & $0(0)$ & $79(5.2)$ \\
\hline Unknown drugs & $1(0.7)$ & $3(0.6)$ & $24(3.4)$ & $2(1.4)$ & $1(3)$ & $31(2)$ \\
\hline Food, fish and plant poisoning & $0(0)$ & $9(1.8)$ & $9(1.3)$ & $0(0)$ & $0(0)$ & $18(1.2)$ \\
\hline Animal poisoning & $3(2)$ & $4(0.8)$ & $6(0.9)$ & $4(2.8)$ & $0(0)$ & $17(1.1)$ \\
\hline None opioid analgesics & $5(3.3)$ & $8(1.6)$ & $4(0.6)$ & $0(0)$ & $0(0)$ & $17(1.1)$ \\
\hline Gases & $0(0)$ & $1(0.2)$ & $4(0.6)$ & $7(4.9)$ & $4(12.1)$ & $16(1.1)$ \\
\hline Miscellaneous drugs ${ }^{\dagger}$ & $0(0)$ & $3(0.6)$ & $1(0.1)$ & $0(0)$ & $2(6.1)$ & $6(0.4)$ \\
\hline $\begin{array}{l}\text { Miscellaneous } \\
\text { non-medicinal agents }{ }^{\dagger \dagger}\end{array}$ & $7(4.7)$ & $0(0)$ & $3(0.4)$ & $0(0)$ & $0(0)$ & $10(0.7)$ \\
\hline Total & 150 & 494 & 700 & 144 & 33 & $1521(100)$ \\
\hline
\end{tabular}

$N$ : number, (\%): percentage by PSS grade , ${ }^{\dagger}$ : included iron supplements (2 cases), primperan (one case), colchicine (5 cases) and anticoagulants (2 cases). ${ }^{t \prime}$ : included: hypernatremia (2 cases), paraphenylene Diamine (one case), silver nitrate (one case), dormex (one case) and cyanide (one case). 
Table (4): Vital signs of the paediatric poisoned patients admitted to PCC, Ain Shams University Hospitals during the year 2012:

\begin{tabular}{|c|c|c|c|c|}
\hline \multicolumn{2}{|c|}{ Vital signs' findings } & Number & Percentage & Most frequent cause \\
\hline \multirow{4}{*}{$\begin{array}{l}\text { Heart rate } \\
\text { (beats per minute) }\end{array}$} & Normal & 1095 & 72 & \\
\hline & Tachycardia & 377 & 24.8 & $\begin{array}{l}\text { Theophylline } \\
\text { (182 cases, } 48.3 \%)\end{array}$ \\
\hline & Bradycardia & 38 & 2.5 & $\begin{array}{l}\text { Digoxin } \\
\text { (11 cases, 28.9\%) }\end{array}$ \\
\hline & Arrest & 11 & 0.7 & $\begin{array}{l}\text { OPC } \\
\text { (5 cases, } 45.5 \% \text { ) }\end{array}$ \\
\hline \multirow{3}{*}{$\begin{array}{l}\text { Blood pressure } \\
\text { in } \mathrm{mmHg}\end{array}$} & Normal & 1452 & 95.5 & \\
\hline & Hypotension & 45 & 2.9 & $\begin{array}{l}\text { Theophylline } \\
\text { (12 cases, } 26.7 \% \text { ). }\end{array}$ \\
\hline & Shock & 24 & 1.6 & $\begin{array}{l}\text { OPC } \\
\text { ( } 7 \text { cases, } 29.2 \%)\end{array}$ \\
\hline \multirow{4}{*}{$\begin{array}{l}\text { Respiratory rate } \\
\text { (breaths per minute) }\end{array}$} & Normal & 1435 & 94.4 & \\
\hline & Tachypnea & 44 & 2.8 & $\begin{array}{l}\text { Kerosene } \\
(33 \text { cases, } 75 \%)\end{array}$ \\
\hline & Bradypnea & 21 & 1.4 & $\begin{array}{l}\text { Carbon monoxide } \\
(10 \text { cases, } 47.6 \%)\end{array}$ \\
\hline & Apnea & 21 & 1.4 & $\begin{array}{l}\text { OPC } \\
\text { (8 cases, } 38.1 \%)\end{array}$ \\
\hline \multirow{3}{*}{$\begin{array}{l}\text { Temperature } \\
\left({ }^{\circ} \mathrm{C}\right)\end{array}$} & Normal & 1338 & 87.9 & \\
\hline & Hypothermia & 100 & 6.6 & $\begin{array}{l}\text { (OPC) } \\
\text { (49 cases, } 49 \%) .\end{array}$ \\
\hline & Hyperthermia & 83 & 5.5 & $\begin{array}{l}\text { Corrosives } \\
(21 \text { cases, } 25.3 \%)\end{array}$ \\
\hline \multirow{5}{*}{$\begin{array}{l}\text { Consciousness } \\
\text { level }\end{array}$} & Come grade 0 & 1199 & 78.9 & \\
\hline & Coma grade I & 213 & 14 & \multirow{4}{*}{$\begin{array}{l}\text { CNS drugs } \\
(163 \text { cases, } 50.6 \%)\end{array}$} \\
\hline & Coma grade II & 79 & 5.2 & \\
\hline & Coma grade III & 11 & 0.7 & \\
\hline & Coma grade IV & 19 & 1.2 & \\
\hline \multicolumn{2}{|l|}{ Total } & 1521 & 100 & \\
\hline
\end{tabular}


Table (5): Gastro-intestinal, neurological, respiratory, genitor-urinary and cutaneous manifestations of the paediatric poisoned patients admitted to PCC, Ain Shams University Hospitals during the year 2012:

\begin{tabular}{|c|c|c|c|c|}
\hline \multicolumn{3}{|c|}{ Clinical findings } & Number & Percentage (\%) \\
\hline \multirow[t]{7}{*}{ Gastro-intestinal } & \multicolumn{2}{|c|}{ Nausea And Vomiting } & 754 & 75.7 \\
\hline & \multicolumn{2}{|c|}{ Diarrhea } & 116 & 11.7 \\
\hline & \multicolumn{2}{|c|}{ Abdominal Colic } & 44 & 4.4 \\
\hline & \multicolumn{2}{|c|}{ Dysphagia } & 39 & 3.9 \\
\hline & \multicolumn{2}{|c|}{ Hematemesis } & 32 & 3.2 \\
\hline & \multicolumn{2}{|c|}{ Melena } & 11 & 1.1 \\
\hline & \multicolumn{2}{|l|}{ Total } & 996 & 100 \\
\hline \multirow[t]{12}{*}{ Neurological } & \multirow[t]{2}{*}{ Pupils } & Constricted & 295 & 19.4 \\
\hline & & Dilated & 71 & 4.6 \\
\hline & \multicolumn{2}{|c|}{ Fasciculations } & 98 & 28.4 \\
\hline & \multicolumn{2}{|c|}{ Motor paresis } & 65 & 18.8 \\
\hline & \multicolumn{2}{|c|}{ Agitation } & 50 & 14.5 \\
\hline & \multicolumn{2}{|c|}{ Disequilibrium } & 34 & 9.9 \\
\hline & \multicolumn{2}{|c|}{ Seizures } & 30 & 8.7 \\
\hline & \multicolumn{2}{|c|}{ Hallucinations } & 29 & 8.4 \\
\hline & \multicolumn{2}{|c|}{ Extrapyramidal } & 27 & 7.8 \\
\hline & \multicolumn{2}{|c|}{ Ataxia } & 6 & 1.7 \\
\hline & \multicolumn{2}{|c|}{ Sensory deficit } & 6 & 1.7 \\
\hline & \multicolumn{2}{|l|}{ Total } & 345 & 100 \\
\hline \multirow[t]{7}{*}{ Respiratory } & \multicolumn{2}{|c|}{ Coarse Crepitations } & 89 & 42.2 \\
\hline & \multicolumn{2}{|c|}{ Respiratory Distress } & 58 & 27.5 \\
\hline & \multicolumn{2}{|l|}{ Cough } & 20 & 9.5 \\
\hline & \multicolumn{2}{|c|}{ Rhonchi } & 20 & 9.5 \\
\hline & \multicolumn{2}{|c|}{ Pulmonary Edema } & 14 & 6.6 \\
\hline & Stridor & & 10 & 4.7 \\
\hline & Total & & 211 & 100 \\
\hline Genito-Urinary & Urinary & Incontinence & 187 & 96.9 \\
\hline & Urinary & Retention & 3 & 1.6 \\
\hline & Priapis & & 3 & 1.6 \\
\hline & Total & & 193 & 100 \\
\hline Cutaneous & Sweati & & 98 & 51.3 \\
\hline & Cyanos & & 47 & 24.6 \\
\hline & Flushin & - Erythema & 16 & 8.4 \\
\hline & Edema & & 13 & 6.8 \\
\hline & Bullae & & 8 & 4.2 \\
\hline & Pallor & & 7 & 3.7 \\
\hline & Pain & & 2 & 1.0 \\
\hline & Total & & 191 & 100 \\
\hline
\end{tabular}

Table (6): Results of serum electrolytes and blood sugar levels tests for paediatric poisoned patients admitted to PCC Ain Shams University Hospitals during the year 2012:

\begin{tabular}{|c|c|c|c|c|}
\hline \multicolumn{2}{|c|}{ Laboratory test } & Number & Percentage $(\%)$ & Most frequent cause \\
\hline \multirow{4}{*}{ Random blood sugar (RBG) } & Normal & 1075 & 80.9 & \\
\hline & Hypoglycemia & 194 & 14.6 & $\begin{array}{l}\text { Pesticides } \\
(38 \text { cases, } 19.6 \%)\end{array}$ \\
\hline & Hyperglycemia & 60 & 4.5 & $\begin{array}{l}\text { Theophylline } \\
\text { (16 cases, } 26.7 \% \text { ) }\end{array}$ \\
\hline & Total & 1329 & 100 & \\
\hline \multirow{4}{*}{ Sodium $\left(\mathrm{Na}^{+}\right)$serum level } & Normal & 1459 & 95.9 & \\
\hline & Hyponatremia & 60 & 3.9 & $\begin{array}{l}\text { Pesticides } \\
(35 \text { cases, } 58 \%) \text {. }\end{array}$ \\
\hline & Hypernatremia & 2 & 0.2 & Salty water \\
\hline & Total & 1521 & 100 & \\
\hline \multirow{3}{*}{ Potassium $\left(\mathrm{K}^{+}\right)$serum level } & Normal & 476 & 31.2 & \\
\hline & Hypokalemia & 697 & 45.8 & $\begin{array}{l}\text { Pesticides } \\
(171 \text { cases, } 4.5 \%)\end{array}$ \\
\hline & Total & 1521 & 100 & \\
\hline
\end{tabular}


Table (7): Findings of blood gases tests that had been done for the pediatric poisoned patients admitted to PCC, Ain Shams University Hospitals during the year 2012:

\begin{tabular}{|l|l|l|l|}
\hline \multicolumn{1}{|c|}{ Findings } & N & \% & Most frequent cause \\
\hline Normal & 167 & 50.7 & \\
\hline Metabolic acidosis & 75 & 22.7 & Pesticides (22 cases, 29.3\%) \\
\hline Respiratory acidosis & 45 & 13.6 & Tramadol (20 cases, 44.4\%) \\
\hline Respiratory alkalosis & 43 & 13.0 & Theophylline (26 cases, 57.8\%) \\
\hline Total & 330 & 100 & \\
\hline
\end{tabular}

Table (8): Specific toxicological test for diagnosis of acute poisoning in paediatric poisoned patients admitted to PCC, Ain Shams University Hospitals during the year 2012:

\begin{tabular}{|c|c|c|}
\hline Findings & Number & Percentage (\%) \\
\hline Pseudocholinesterase level in blood & 351 & 72.1 \\
\hline Screening test for tramadol & 50 & 1.3 \\
\hline Digoxin level in blood & 18 & 3.7 \\
\hline Carbamazepine level in blood & 15 & 3.1 \\
\hline Paracetamol level in blood & 12 & 2.5 \\
\hline Screening test for benzodiazepines & 11 & 2.3 \\
\hline Screening test for cannabis & 10 & 2.1 \\
\hline Screening test for phenothiazines & 9 & 1.8 \\
\hline Salicylates level in blood & 8 & 1.6 \\
\hline Screening test for opiates & 3 & 0.6 \\
\hline Total & 487 & 100 \\
\hline
\end{tabular}

Table (9): Abnormalities of ECG of the paediatric poisoned patients, admitted to PCC, Ain Shams University

Hospitals, during the year 2012:

\begin{tabular}{|l|c|c|}
\hline Electrocardiograph (ECG) abnormality & Number & Percentage (\%) \\
\hline Sinus Tachycardia & 318 & 88.4 \\
\hline Sinus Bradycardia with sagging in ST segment & 12 & 3.4 \\
\hline Ventricular arrhythmias & 10 & 2.8 \\
\hline Sinus bradycardia & 8 & 2.3 \\
\hline Nodal rhythm & 3 & 0.9 \\
\hline Second degree heart block & 2 & 0.5 \\
\hline Ischemic changes & 2 & 0.5 \\
\hline Dropped beats & 2 & 0.5 \\
\hline Atrial arrhythmias & 2 & 0.5 \\
\hline Third degree heart block & 1 & 0.2 \\
\hline Total & 360 & 100 \\
\hline
\end{tabular}

Table (10): Chi-square statistical analysis comparing the place of admission between the age groups of the pediatric poisoned patients admitted to PCC, Ain Shams University Hospitals during the year 2012:

\begin{tabular}{|c|c|c|c|c|c|}
\hline \multirow{2}{*}{ Age group $\quad$ Admission place } & \multicolumn{2}{|l|}{ Inpatient } & \multicolumn{2}{|l|}{ (ICU) } & \multirow[t]{2}{*}{ Total } \\
\hline & Number & $(\%)$ & Number & $(\%)$ & \\
\hline Infants $(<1 \mathrm{Y})$. & 24 & 85.7 & 4 & 14.3 & 28 \\
\hline Toddlers $(1-<3$ Y.) & 259 & 77.5 & 75 & 22.5 & 334 \\
\hline Preschool age $(3-<6$ Y.) & 166 & 77.6 & 48 & 22.4 & 214 \\
\hline School age $(6-<12$ Y.) & 78 & 78 & 22 & 22 & 100 \\
\hline Adolescents $(12-18$ Y.) & 735 & 87 & 110 & 13 & 845 \\
\hline Total & 1262 & 83 & 259 & 17 & 1521 \\
\hline Chi-square & \multicolumn{5}{|c|}{$\mathrm{X}^{2}: 28.634$} \\
\hline & \multicolumn{5}{|c|}{ P-value: $<0.001 *$} \\
\hline
\end{tabular}

Y: year(s), N: Number, (\%): Percentage by age group, *: statistically significant. 
Table (11): Durations of hospital stay in relation to grades of PSS of the pediatric poisoned patients admitted to PCC, Ain Shams University Hospitals during the year 2012 in relation to PSS:

\begin{tabular}{|c|c|c|c|c|c|c|c|c|c|}
\hline \multirow{2}{*}{$\begin{array}{r}\text { Hospital stay } \\
\text { (in days) }\end{array}$} & \multicolumn{2}{|l|}{$<1$} & \multicolumn{2}{|l|}{$1-3$} & \multicolumn{2}{|c|}{$4-<7$} & \multicolumn{2}{|c|}{$>7$} & \multirow[t]{2}{*}{ Total } \\
\hline & $\mathbf{N}$ & $(\%)$ & $\mathbf{N}$ & $(\%)$ & $\mathbf{N}$ & $(\%)$ & $\mathbf{N}$ & $(\%)$ & \\
\hline 0 & 120 & 027 & 11 & 73 & , & 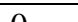 & , & 0 & 150 \\
\hline 1 & 422 & 854 & 72 & 146 & 0 & 0 & 0 & 0 & 494 \\
\hline 2 & 525 & 75 & 169 & 24.1 & 4 & 0.6 & 2 & 0.3 & 700 \\
\hline 3 & 50 & 34.7 & 65 & 45.1 & 20 & 13.9 & 9 & 6.3 & 144 \\
\hline 4 & 5 & 15.2 & 9 & 27.3 & 10 & 30.3 & 9 & 27.3 & 33 \\
\hline Total & 1141 & 75 & 326 & 21.4 & 34 & 2.2 & 20 & 1.3 & 1521 \\
\hline
\end{tabular}

N: Number, \%: Percentage by PSS grade.

Table (12): The poisonous agents responsible for prolonged duration of hospital stay ( $\geq 4$ days) in relation to PSS of the pediatric poisoned patients admitted to PCC, Ain Shams University Hospitals during the year 2012 in relation to PSS:

\begin{tabular}{|c|c|c|c|c|c|c|c|}
\hline & 2 & & 3 & & 4 & & Total \\
\hline Poisonous agent & $\mathbf{N}$ & $\%$ & $\mathbf{N}$ & $\%$ & $\mathbf{N}$ & $\%$ & \\
\hline Corrosive & 4 & 25 & 8 & 50 & 4 & 25 & 16 \\
\hline Pesticides & 1 & 7.7 & 9 & 69.2 & 3 & 23.1 & 13 \\
\hline Carbon monoxide & 0 & 0 & 3 & 42.9 & 4 & 57.1 & 7 \\
\hline Antiepileptic & 0 & 0 & 1 & 20 & 4 & 80 & 5 \\
\hline Tramadol & 0 & 0 & 1 & 33.3 & 2 & 66.7 & 3 \\
\hline Antipsychotics & 1 & 33.3 & 1 & 33.3 & 1 & 33.3 & 3 \\
\hline Kerosene & 0 & 0 & 1 & 50 & 1 & 50 & 2 \\
\hline Calcium channel blockers & 0 & 0 & 1 & 100 & 0 & 0 & 1 \\
\hline Theophylline & 0 & 0 & 2 & 100 & 0 & 0 & 2 \\
\hline Paraphenylene Diamine (PPD) & 0 & 0 & 1 & 100 & 0 & 0 & 1 \\
\hline Unknown & 0 & 0 & 1 & 100 & 0 & 0 & 1 \\
\hline Total & 6 & 11.1 & 29 & 53.7 & 19 & 35.2 & 54 \\
\hline
\end{tabular}

Table (13): Poisonous agents in cases who required MV in pediatric poisoned patients admitted to PCC, Ain Shams University Hospitals, during the year 2012:

\begin{tabular}{|l|c|c|}
\hline \multicolumn{3}{|c|}{ Mechanical ventilation (MV) } \\
\hline Poisonous agent & Number & Percentage (\%) \\
\hline Pesticides & 16 & 25.4 \\
\hline Substances Of Abuse & 10 & 15.9 \\
\hline Gases & 10 & 15.9 \\
\hline CNS Drugs & 9 & 14.3 \\
\hline Chemicals & 5 & 7.9 \\
\hline Rat Poison & 3 & 4.8 \\
\hline Animal Poisoning & 3 & 4.8 \\
\hline Cardiopulmonary & 3 & 4.8 \\
\hline Unknown Drugs & 2 & 3.1 \\
\hline Hypernatremia & 2 & 3.1 \\
\hline Total & 63 & 100 \\
\hline
\end{tabular}


Table (14): Supportive treatments received by the pediatric poisoned patients admitted to PCC, Ain Shams University Hospitals during the year 2012:

\begin{tabular}{|l|c|c|}
\hline \multicolumn{1}{|c|}{ Supportive Treatment } & Number & Percentage (\%) \\
\hline IV fluids & 1521 & 100 \\
\hline $\mathrm{H}_{2}$ Blockers/PPIs/Anti-Emetics & 856 & 56.3 \\
\hline Antibiotics & 169 & 11.1 \\
\hline Sedative Hypnotics/Anticonvulsants & 148 & 9.7 \\
\hline Steroids & 130 & 8.5 \\
\hline Mechanical Ventilation & 63 & 4.1 \\
\hline Inotropic & 26 & 1.7 \\
\hline Mannitol & 22 & 1.4 \\
\hline Sodium Bicarbonate & 18 & 1.2 \\
\hline Anti-histaminics & 8 & 0.5 \\
\hline Blood transfusion & 2 & 0.1 \\
\hline
\end{tabular}

(\%): Percentage of the number of admitted cases. ${ }^{\dagger}$ PPIs: proton pump inhibitors, IV: intravenous.

Table (15): Antidotes given to the children admitted to the PCC, Ain Shams University Hospitals during the year 2012:

\begin{tabular}{|l|c|c|}
\hline Antidotes & Number & Percentage (\%) \\
\hline Atropine & 202 & 59.7 \\
\hline Atropine and Oximes & 91 & 26.9 \\
\hline Naloxone & 11 & 3.3 \\
\hline N-acetyl cysteine & 11 & 3.3 \\
\hline Scorpion antivenom & 10 & 3 \\
\hline Oximes & 6 & 1.8 \\
\hline Snake antivenom & 4 & 1.2 \\
\hline Desferal & 2 & 0.6 \\
\hline Flumazenil & 1 & 0.2 \\
\hline Total & 338 & 100 \\
\hline
\end{tabular}

Table (16): Case fatality rates of acute poisoning in pediatric poisoned patients admitted to PCC, Ain Shams University Hospitals during the year 2012:

\begin{tabular}{|l|c|c|c|}
\hline Causative agent & Deaths & Total number of cases & Case fatality rate \\
\hline Organophosphates & 8 & 220 & 3.6 \\
\hline CNS Drugs & 7 & 205 & 3.4 \\
\hline Chemicals & 6 & 119 & 5.0 \\
\hline Carbon monoxide & 4 & 14 & 28.6 \\
\hline Phosphide & 2 & 134 & 1.5 \\
\hline Tramadol & 2 & 88 & 2.3 \\
\hline Hypernatremia & 2 & 2 & 100 \\
\hline Digoxin & 1 & 47 & 2.1 \\
\hline Unknown & 1 & 31 & 3.2 \\
\hline
\end{tabular}




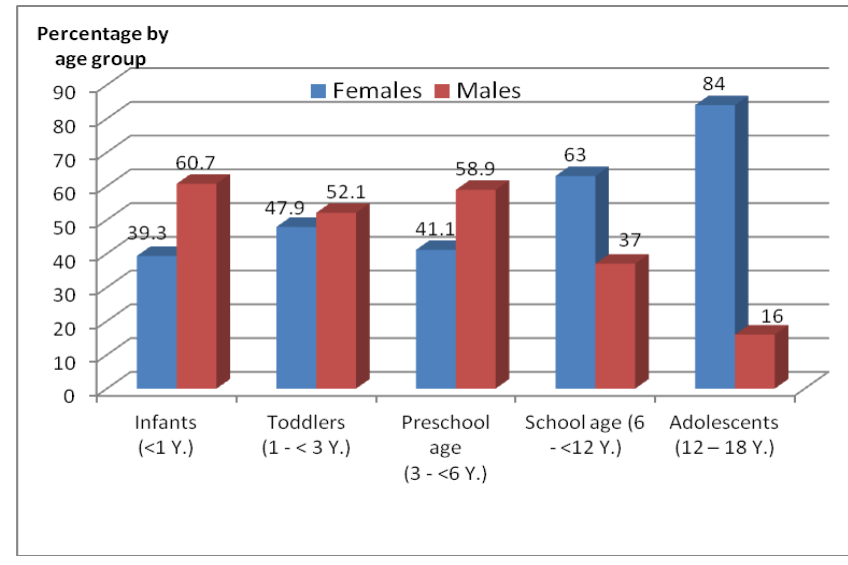

Figure (1): Gender distribution in age groups of poisoned children, admitted to PCC, Ain Shams University Hospitals during the year 2012

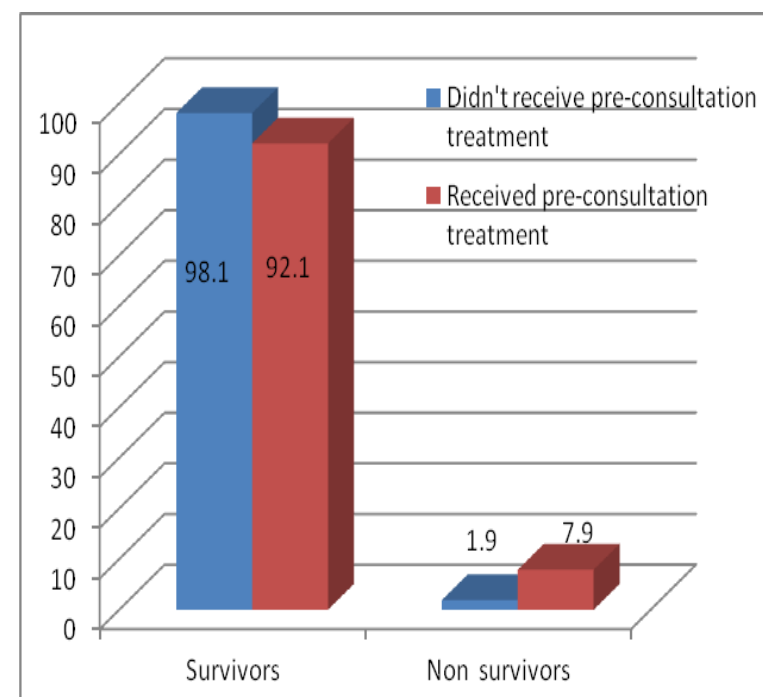

Figure (3): Mortalities in pediatric poisoned patients admitted to PCC, Ain Shams University Hospitals during the year 2012 in relation to pre-consultation treatment.

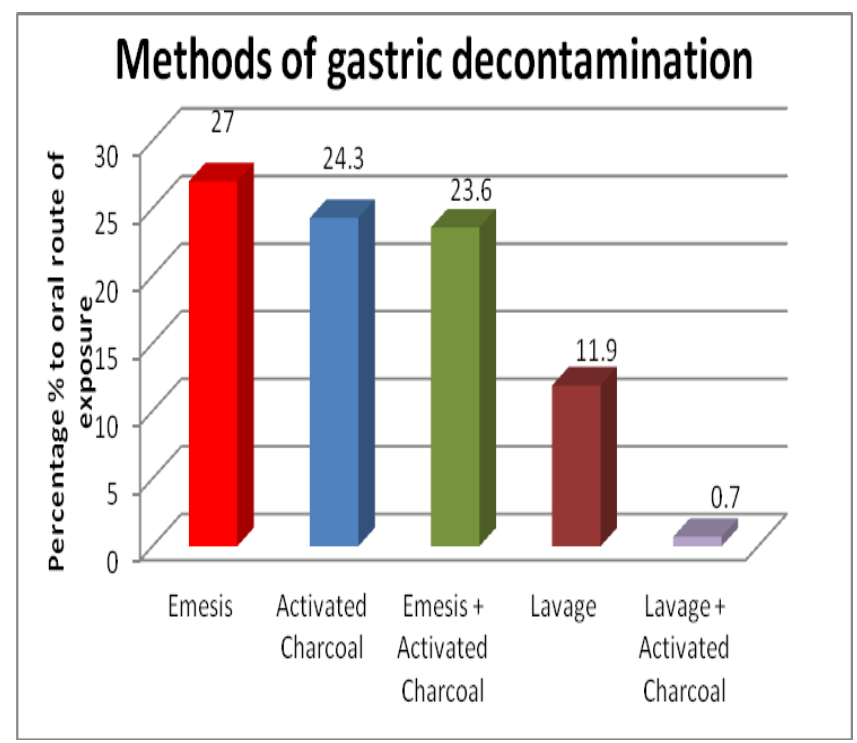

Figure (5): Methods of gastric decontamination in children with oral route of exposure admitted to PCC, Ain Shams University Hospitals during the year 2012.

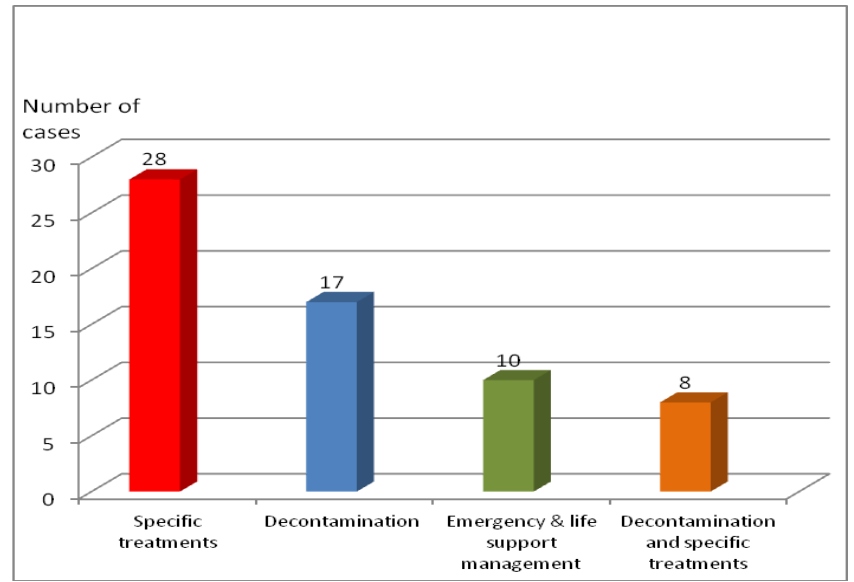

Figure (2): Pre-consultation treatment in the pediatric poisoned patients admitted to PCC, Ain Shams University Hospitals during the year 2012.

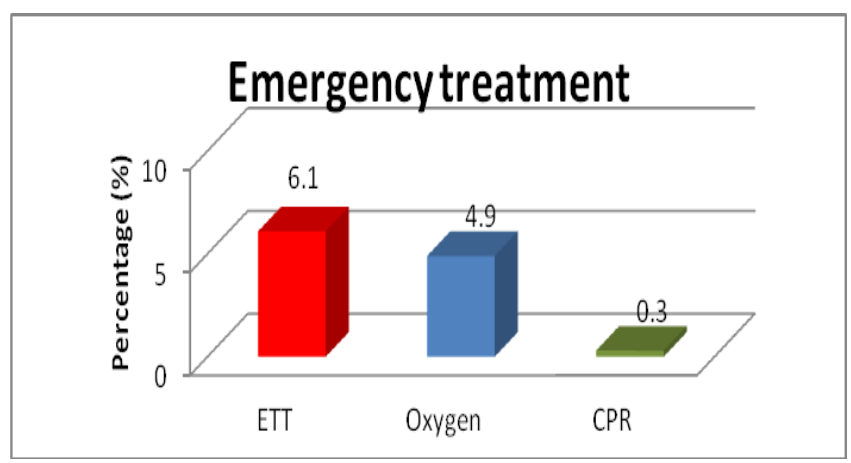

Figure (4): Emergency treatments received by the poisoned children admitted to PCC, Ain Shams University Hospitals during the year 2012.

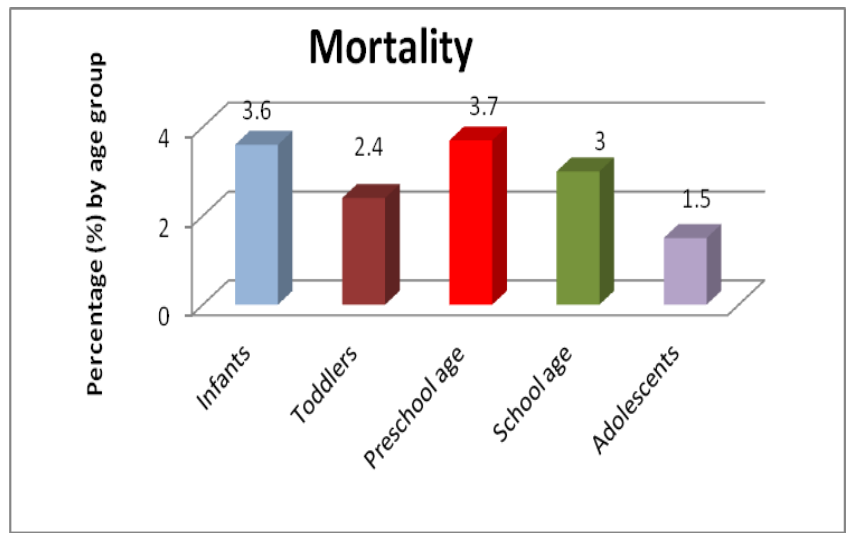

Figure (6): Percentage of mortality in age groups of pediatric poisoned patients admitted to $\mathrm{PCC}$, Ain Shams University Hospitals during the year 2012. 


\section{Discussion}

Hazard of poisoning by variable agents have been increased due to several factors such as rapid industrialization and exposure to hazardous chemical products, introduction of newer range of drugs for treatment, massive use of pesticides in agriculture and unhealthy dietary habits. (Kiran et al., 2008). Children are particularly at risk because of their curious and exploratory behavior (Vasavada and Desai, 2013).

This study showed a significant increase of the median of delay time in non-survivors than that in survivors. This agreed with Ozdemir et al., (2012) and Ramesha et al, (2009) who studied childhood poisoning in Turkey and India respectively and found an association between prolonged delay time and mortality as majority of deaths occurred in patients presented to the hospital with a delay time exceeding 8 hours.

The duration of pre-hospitalization period was found as a strong predictive factor of severity of poisoning. This was explained by the fact that, delayed treatment allows the suspected initial peak blood level of poison to induce irreversible tissue damage (Sam et al., (2009).

The current study found significant increase of mortality in patients who received pre-consultation treatment than that in patients who didn't receive any treatment before arrival to the PCC.

This may be due to improper pre-consultation management as found by Veale et al. (2013) who reported that, poisoning had been misdiagnosed in prereferral hospitals in South Africa as organophosphate poisoning and atropine wrongly administered as the antidote in amitraz, glyphosate and aluminium phosphide poisoning.

The same findings were reported by El Masry and Azab, (2013) who studied inappropriate management and transfer of referred patients to the PCC, Ain Shams University Hospitals and found that, 52 cases received improper management that was either incorrect medications (40 cases) or faulty decontamination or resuscitation maneuvers (12 cases).

This study revealed faulty management in the form of induction of emesis by salty water that was given to $2.6 \%$ of the included cases (39 cases). The percentage of mortality among these cases was $7.7 \%$. Similar findings were reported by El Masry and Azab, (2013) who found that, 9\% of the cases with inappropriate management measures were given salty water for induction of emesis. Death in these cases is most probably due to hypernatremia which is a serious complication of intake of salty water (Su et al., 2010).

In this study, most of the included cases were admitted in the inpatient section. This agreed with Taghaddosinejad et al. (2012) who reported that, ICU admission is strictly limited to severe and life threatening poisoning.

The current study found most cases were presented with normal vital signs. The most common abnormalities were tachycardia, hypotension, tachypnea and hypothermia. This agreed with Alazab, (2012) who studied acute poisoning in children admitted to a University Hospital in Egypt and found the majority of cases presented with normal vital signs. He found that, hypotension, followed by tachypnea, was the most common abnormality in vital functions.

Theophylline was the most frequent cause of tachycardia and hypotension in this study while kerosene was the most frequent cause of tachypnea and organophosphorous compounds (OPC) were the most frequent cause of hypothermia (49 cases, 49\%).

Theophylline may cause sinus tachycardia and tachypnea through its sympathomimetic action and positive chronotropic effect (this was evident in ECG, as sinus tachycardia was the most common abnormality). It may also cause hypotension due to increased beta-2 receptor stimulation (Aggarwal, 2012). Tachycardia and tachypnea can occur as a physiologic response to catecholamine release due to stress and anxiety (Viswanathan and Kollef, 2012).

Poisoned cases with kerosene are commonly presented with tachypnea and signs of respiratory distress due to chemical pneumonitis (Osterhoudt et al., 2006).

(OPC) may cause initial hypothermia due to direct CNS administration of cholinergic agonists in the region of the hypothalamus or cerebral ventricles (Moffatt et al., 2010).

This study found that, cases presented with constricted pupils were more frequent than those presented with dilated pupils. This may be due to high prevalence of pesticides (mainly OPC and carbamates) as they were the most common non-medicinal poisonous agent in the included cases. They are commonly presented with constricted pupils due to the nicotinic action of the excess of acetylcholine in synapses and neuromuscular junctions (Rajendiran et al., 2009).

Mydriasis can result from increased sympathetic stimulation as in cases affected by cocaine, amphetamines and hypoglycemia or from inhibition of muscarinic cholinergic-mediated pupillary constriction secondary to exposure to anticholinergic agents (Perera et al., 2008).

In this study, gastrointestinal manifestations (mainly nausea and vomiting) were the most frequent manifestations among the included cases, followed by neurological manifestations. This agreed with previous studies on poisoned pediatric patients that found the 
common presenting systems symptomatology were digestive and CNS (Bhat et al., 2012 and Shwe et al., 2013).

These can be explained by the prevalence of pesticides as they were the most common agents in the included cases. Vomiting in (OPC) toxicity occurs due to excessive muscarinic activity (Aggarwal, 2012). Neurological manifestations are common with (OPC) and carbamates due to their nicotinic action. The effects of excessive cholinergic stimulation at these sites are similar to that of a depolarizing neuromuscular blocker agent (succinylcholine) initially resulting in fasciculations, hypotonia or weakness (Clark, 2006).

Also, respiratory manifestations are common with insecticide toxicity due to excessive stimulation of muscarinic receptors which leads to bronchorrhea and bronchospasm (Rajendiran et al., 2009). (OPC) toxicity can result in respiratory failure and development of cyanosis due to weakness of the respiratory muscles, paralysis of the respiratory center, bronchospasm and increased bronchial secretion (Prasad, 2010).

In this study, the most common abnormalities found in laboratory investigations were hypoglycemia, hyponatremia and hypokalemia. Pesticides were the most common causes of hypokalemia, hypoglycemia and hyponatremia.

In (OPC), excessive adrenergic influences on metabolism cause glycogenolysis with hyperglycemia and ketosis that are occasionally mistaken for diabetic ketoacidosis. Hypoglycemia can also occur, although the mechanism is unclear (Clark, 2006). Hypoglycemia can be caused by many drugs as beta blockers, oral hypoglycemic drugs and salicylate poisoning hyperinsulinism (Josefson and Zimmerman, 2009).

Significant fluid, sodium and potassium concentration abnormalities commonly occur in the setting of xenobiotic exposure. Gastrointestinal losses may occur in the form of vomiting, diarrhea or bleeding. Renal fluid losses may result from increased glomerular filtration rate (inotropes) or impaired absorption (diuretics). Fluid losses also may occur through the skin as a result of sweating as in cases of sympathomimetics, cholinergics or salicylates poisoning or through the lungs as a result of bronchorrhea as in cholinergics (Charney and Hoffman, 2006).

Hypokalemia can result from acute theophylline toxicity due to influx of extracellular potassium into the intracellular compartment despite normal total body potassium content. Also, it is common finding in patients with toxicity from $\beta$ adrenergic agonist activity (Jones and Alpern, 2014). (OPC) can cause hypokalemia due to excessive vomiting and diarrhea (Balali-Mood and Saber, 2012).

In this study, metabolic acidosis was the most common blood gases' abnormality caused most commonly by pesticides, followed by respiratory acidosis caused mainly by tramadol.
Metabolic acidosis with increased serum lactate levels is commonly noted as a complication of OPC, theophylline, salicylate overdose (Jaiswal et al., 2009 and Mégarbane, 2014).

Respiratory acidosis can be primarily caused by the toxin as a result of central respiratory center depression (as in tramadol and opioid toxicity) (Sood et al., 2010) or a premorbid finding due to loss of respiratory compensation for the metabolic acidosis caused by other toxins as pesticides, theophylline or salicylate toxicity (Pearlman and Gambhir, 2009).

Respiratory alkalosis represents the earliest acid-base abnormality of salicylate and theophylline intoxication due to a direct stimulation of the respiratory center (Liamis et al., 2010).

In this study, most of the included cases were admitted in the inpatient department. This agreed with Veale et al., (2013) who studied poisoning in South Africa where most children were admitted for overnight observation while only $5 \%$ were admitted to the ICU.

This can be explained by the fact that, ICU admission is strictly limited to severe and life threatening poisoning (Taghaddosinejad et al., 2012).

The present study revealed an association between increased severity of poisoning and prolonged duration of hospital stay. This agrees with Paterson et al., (2006) and Sam et al., (2009) who found an association between the increased duration of hospital stay and the significant mortality and morbidity of the poisoned cases.

Corrosives, followed by pesticides were the most frequent cause of prolonged hospitalization in thus study. This can be explained by the fact that, in cases with ingestion of corrosives, hospitalization is indicated for cases with severe or significant manifestation that need intensive care of complications until regaining of oral nutrition (Naik and Vadivelan, 2012).

Regarding the received treatment in the PCC, emergency managements were indicated in small percentage of the cases (11.3\%). Poisoning with pesticides was the most frequent causative agent in cases indicated mechanical ventilation, followed by substances of abuse and poisonous gases. This agreed with El Masry and Tawfik, (2013) who found that, (OPC) was the most frequent cause of poisoning in cases required MV (30\%), followed by tramadol $(16 \%)$

Gut decontamination of ingested poisons was performed in the majority of the cases by induction of emesis by syrup ipecac and administration of activated charcoal. Gastric lavage was done in small percentage of the included cases.

Although there is no evidence from clinical studies that emesis by ipecac improves the outcome of poisoned patient, yet its routine use is still present and 
should be prohibited (Yip et al., 2011) as syrup ipecac may cause aspiration, delay the administration or reduce the effectiveness of activated charcoal, oral antidotes, and whole bowel irrigation.

Therefore, recent guidelines of the European Association of Poisons Centers and Clinical Toxicologists as well as the American Academy of Pediatrics recommended that, ipecac should no longer be used routinely as a treatment strategy for treatment of pediatric poisoning (Chyka et al., 2005).

The wide use of ipecac in the PCC for gastric decontamination may be due to limited resources and unavailability of disposable gastric lavage tubes in the market in Egypt which makes ipecac an easier and safer method for gastric decontamination.

On the other hand, activated charcoal is a universal antidote for the majority of poisons (Michael, 2007). Yet, it is contraindicated after the ingestion of corrosive substances, surfactants, or liquid hydrocarbons, and whenever the respiratory tract has not been protected (by intubation) (Chyka et al., 2005).

Symptomatic treatments were given to all of the included cases in the present study. Maintenance IV fluids, followed by medications for treatment of gastric irritation were the most frequent supportive treatment given to the admitted cases. Similar findings were reported by previous studies on childhood poisoning which found that, treatment in most of the cases was non-specific, including general decontamination and supportive-symptomatic therapy (Sahin et al., 2011, Müller and Desel, 2013).

Atropine, followed by oximes, was the most common antidote used in treatment of the included cases in this study. This is can be explained by high prevalence of poisoning by (OPC).

Regarding cases' outcome, this study found the percentage of mortality in the included cases $2.2 \%$. Preschool age group had the greatest percentage of mortality while adolescents group had the smallest percentage.

Similar findings were reported by Bhat et al. (2012) who studied the profile of poisoning in children and adolescent in India and found the greatest mortality ratio in the preschool age group. Also, Haghighat et al., (2013) reported that, acute poisoning in both adults and children is often associated with morbidity rather than mortality. Half of mortalities were in the preschool age group with the highest mortality ratio.

Small proportion of mortality in adolescents group may be explained by predominance of suicidal poisoning in this group since most cases of suicidal attempts aim to draw attention rather than to inflict real harm or cause death. This is of course achieved by taking less toxic drugs in smaller amounts (Ozdemir et al., 2012).

(OPC) was the most frequent cause of mortalities, followed by CNS drugs. Hypernatremia had the greatest case fatality rate, followed by carbon monoxide poisoning. These findings are in accordance with Malangu and Ogunbanjo, (2009) and Haghighat et al., (2013) who found that, (OPC) accounted for the largest proportion of deaths in acutely poisoned cases

Hypernatremia occurred as a complication of use of salty water for induction of emesis and was found to have the highest case fatality rate. It is known as a fatal complication as it results in brain edema and permanent brain damage (Bockenhauer et al., 2010).

\section{Conclusions}

Delay time was significantly increased in nonsurvivors than survivors. Faulty management in the form of induction of emesis by salty water intake was found in $2.6 \%$ of the cases; $7.7 \%$ of these cases were died. Most cases presented with normal vital signs and gastrointestinal manifestations (mainly nausea and vomiting) were the most frequent manifestations in the cases. Hypoglycemia, hyponatremia, hypokalemia and metabolic acidosis were the most common abnormalities found in laboratory investigations. Sinus tachycardia was the most common abnormality found in ECG

Most of the included cases in this study were admitted in the inpatient section (83\%). The majority of the cases $(75 \%)$ were hospitalized for short period that didn't exceed 24 hours. Higher PSS was associated with longer hospital stay periods.

Emergency managements were indicated in $11 \%$ of the cases and procedures for gastric decontamination were done for 1296 cases $(87.3 \%)$. All cases received supportive and symptomatic treatments. Atropine was the most frequent antidote that was used either alone or with toxogonin.

The overall mortality ratio was $2.2 \%$. Preschool age group had the greatest mortality ratio (3.7\%), followed by infant group (3.6\%). Hypernatremia had the highest case fatality rate (100 $\%)$, followed by $\mathrm{CO}$ poisoning $(28.6 \%)$.

\section{Recommendations}

It is important to increase the public awareness of the first aid measures in childhood poisoning to avoid complications of faulty measures as induction of emesis by salty water. Also, it is important to increase public awareness of the services of the information center in the PCC as it presents guidance about management of poisoned cases.

Ongoing childhood poisoning surveillance is needed to track its effects and risk factors and to monitor the impact of appropriate interventions

\section{References}

Aggarwal, P (2012): Syndromic approach to poisoning. In ICU Protocols: A stepwise approach. Chawla R and Todi S (edits), Springer, India: 553-7. 
Alazab RM (2012): Determinants of acute poisoning among children (1-60) months old at a poisoning unit of a university hospital, Egypt, are employed mothers a risk factor? Retrospective cohort study. Journal of American Science; 8(9): 1107-17.

Aqeel M, Munir A and Khan A, (2009): Pattern and frequency of acute poisoning in children. Pak J Med Sci; 25(3): 479-83.

Balali-Mood M and Saber H, (2012): Recent advances in the treatment of organophosphorus poisoning. Iran J Med Sci; 37(2): 75-91.

Bhat NK, Dhar M, Ahmad S et al., (2012): Profile of poisoning in children and adolescents at a North Indian tertiary care centre. JIACM; 13(1): 37-42.

Bockenhauer D, Van'T Hoff W, Dattani M et al., (2010): Secondary nephrogenic diabetes insipidus as a complication of inherited renal diseases. Nephron, Physiology; 116(4): 23-9.

Chadha IA (2003): Poisoning. Ind J Anaesth; 47(5): 402-11.

Charney AN and Hoffman RS, (2006): Fluid, electrolyte and acid base principles. In: Goldfrank's Toxicologic Emergencies ( $8^{\text {th }}$ ed.). Flomenbaum NE, Goldfrank LR, Hoffman RS., et al (edits), McGraw-Hill Companies, New York: 278-95.

Chyka PA, Seger D, Krenzelok EP et al., (2005): American Academy of Clinical Toxicology. European Association of Poisons Centres and Clinical Toxicologists: Position paper: Singledose activated charcoal. Clin Toxicol (Phila); 43: 61-87.

Clark RF, (2006): Insecticides: Organophsphorus insecticides and carbamates In Goldfrank's Toxicologic Emergencies ( $8^{\text {th }}$ ed.). Flomenbaum NE, Goldfrank LR, Hoffman RS, Ann HM et al. (edits), McGraw-Hill Companies, New York:1498-512.

El Masry MK and Azab SM, (2013): Inappropriate management and transfer of cases with acute poisoning referred to poisoning treatment center-Ain Shams University-Cairo. Egyptian Journal of Forensic Sciences; 3(1): 1-7.

El Masry MK and Tawfik HM, (2013): 2011 Annual Report of the Poison Control Centre of Ain Shams University Hospital, Cairo, Egypt. Ain Shams Journal of Forensic Medicine and Clinical Toxicology; 20: 10-7.

Haghighat M, Moravej H and Moatamedi M, (2013): Epidemiology of pediatric acute poisoning in Southern Iran: a hospital based study. Bull Emerg Trauma; 1(1): 28-33.
Holder Y, Matzopoulos R and Smith N, (2008): Poisoning. In: World Report on Child Injury Prevention. Holder M, Oyegbite K, OzanneSmith J et al. (edits), (Geneva, Switzerland): World Health Organization and UNICEF. Available online at:

http://whqlibdoc.who.int/publications/2008/978924156 3574_eng.pdf?frbrVersion=28

Hutchison JS, Ward RE, Lacroix J et al., (2008): Hypothermia therapy after traumatic brain injury in children. New England Journal of Medicine,; 358(23): 2447-56.

Jaiswal S, Verma RK and Tewari N, (2009): Aluminum phosphide poisoning: Effect of correction of severe metabolic acidosis on patient outcome. Indian journal of critical care medicine: peer-reviewed, official publication of Indian Society of Critical Care Medicine; 13(1): 21-4.

Jones MA and Alpern ER, (2014): A 16-month-old girl with irritability after ingesting white pills. Pediatric emergency care; 30(1): 69-71.

Josefson J and Zimmerman D, (2009): Hypoglycemia in the emergency department. Clinical Pediatric Emergency Medicine; 10(4): 285-91.

Kail RV, (2004): Cognitive development includes global and domain specific processes. MerrillPalmer Quarterly; 50(4): 445-55.

Kiran N, Rani RH and Prakash VJ, (2008): Pattern of poisoning reported at south Indian tertiary care hospital. Indian Journal of Forensic Medicine \&Toxicology; 2(2): 17-9.

Liamis G, Milionis HJ and Elisaf M, (2010): Medication-induced hypophosphatemia: A review. QJM; 103(7): 449-59.

Malangu N and Ogunbanjo GA, (2009): A profile of acute poisoning at selected hospitals in South Africa.South Afr J Epidemiol Infect; 24(2): 14-6.

Mégarbane B, (2014): Toxidrome-based approach to common poisonings. Asia Pacific Journal of Medical Toxicology; 3(1): 2-12.

Michael LR, (2007): Activated charcoal for pediatric poisonings: the universal antidote?. Current opinion in Pediatrics; 19(2): 216-22.

Moffatt A, Mohammed F, Eddleston M et al., (2010): Hypothermia and fever after organophosphorus poisoning in humans- A prospective case series. Journal of Medical Toxicology; 6(4): 379-85.

Müller D and Desel H, (2013): Common causes of poisoning: etiology, diagnosis and treatment. Deutsches Ärzteblatt International; 110(41): 690-700. 
Naik RR and Vadivelan M, (2012): Corrosive Poisoning. Indian Journal of Clinical Practice; 23(3): 131-4

Osterhoudt KC, Burns Ewald M, Shannon M et al., (2006): Toxicologic emergencies. In: Textbook of pediatric emergency medicine (5th ed.), Fleisher, GR, Ludwig, S, Henretig, FM (eds), Lippincott, Williams \& Wilkins, Philadelphia: 951.

Ozdemir R, Bayrakci B, Teksam O, Yalçin, B et al., (2012): Thirty-three-year experience on childhood poisoning. Turk J Pediatr; 54(3): 251-9.

Paterson R, Macleod DC, Thetford D et al., (2006): Prediction of in-hospital mortality and length of stay using an early warning scoring system: clinical audit. Clin Med; 6 (3): 281-4.

Pearlman BL and Gambhir R, (2009): Salicylate Intoxication. Postgraduate Medicine; 121(4): 162-8.

Perera PMS, Shahmy S, Gawarammana I et al., (2008): Comparison of two commonly practiced atropinization regimens in acute organophosphorus and carbamate poisoning, doubling doses vs. ad hoc: a prospective observational study. Human \& Experimental Toxicology; 27(6): 513-8.

Persson H, Sjöberg G, Haines J et al., (1998): Poisoning Severity Score: Grading of acute poisoning. J Toxicology - Clinical Toxicology; 36: 205-13.

Prasad M, (2010): Significance of hypokalemia in acute organophosphorus poisoning. Arch Appl Sci Res; 4: 216-22.

Rajendiran C, Ravi G and Subramanian PT, (2009): Organophosphate, carbamate and rodenticide poisoning in children. Indian Journal of Practical Pediatrics; 11(1): 6-14.

Ramesha KN, Rao KB and Kumar GS, (2009): Pattern and outcome of acute poisoning cases in a tertiary care hospital in Karnataka, India. Indian journal of critical care medicine: peer-reviewed, official publication of Indian Society of Critical Care Medicine; 13(3): 1525.

Randev S, Grover N, Sharma R et al., (2011): Acute poisoning in children: seven year experience at a tertiary care hospital of north India. Current Pediatric Research; 15(1): 65-8.

Sahin S, Carman KB and Dinleyici EC, (2011): Acute poisoning in children; data of a pediatric emergency unit. Iran J Pediatr; 21 (4): 479-84.
Sam KG, Kondabolu K, Pati D, Kamath A, et al., (2009): Poisoning severity score, APACHE II and GCS: effective clinical indices for estimating severity and predicting outcome of acute organophosphorus and carbamate poisoning. Journal of Forensic and Legal Medicine; 16(5): 239-47.

Schafermayer RW, (2012): Pediatric trauma. In: Prehospital Care Pearls and Pitfalls. Pons PT and Markovchick VJ (edits), PMPH, USA: 450-5.

Shwe DD, Toma B, Pate SI et al., (2013): Profile of hospital Admissions of childhood poisoning at a North-central Nigerian tertiary health care centre. Jos Journal of Medicine; 7(2): 5-11.

Sood P, Paul G and Puri S (2010): Interpretation of arterial blood gas. Indian journal of critical care medicine: peer-reviewed, official publication of Indian Society of Critical Care Medicine; 14(2): 57-64.

Su E, Shoykhet M and Bell MJ, (2010): Severe hypernatremia in a hospitalized child: Munchausen by proxy. Pediatric Neurology; 43(4): 270-3.

Taghaddosinejad F, Sheikhazadi A, Yaghmaei A, et al., (2012): Epidemiology and Treatment of Severe Poisoning in the Intensive Care Unit: Lessons from a one-year prospective observational study. J Clin Toxicol; S:1:007. Available online at: http://omicsonline.org/epidemiology-andtreatment-of-severe-poisoning-in-theintensive-careunit-lessons-2161-0495.S1007.pdf

Vasavada H and Desai P, (2013): Clinical profile and outcome of children presenting with poisoning (A Hospital Based Study). NJIRM; 4(4) : 1-7

Veale DJH, Wium CA and Müller GJ, (2013): Toxicovigilance II: A survey of the spectrum of acute poisoning and current practices in the initial management of poisoning cases admitted to South African hospitals. SAMJ: South African Medical Journal; 103(5): 298303.

Viswanathan S and Kollef M, (2012): Cardiac dysrhythmias and conduction abnormalities. In: The Washington manual of critical care ( $2^{\text {nd }}$ ed.). Kollef $\mathrm{M}$ and Isakow $\mathrm{M}$ (edits), Lippincott Williams \& Wilkins, Philadelphia: 139-54.

Yip WL, Ng HW and Tse ML (2011): An epidemiological study of paediatric poisoning in Hong Kong. HK J Paediatr (New Series); 16: $25-31$. 


\section{الملخص العربيى}

الوجهة الاكلينيكية والنتيجة فى الأطفال المرضى المصابين بالتسمم والذين أدخلوا مركز علاج التسمم،

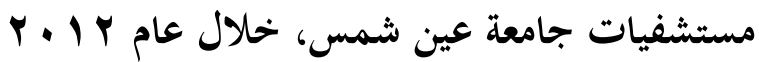

\section{سوسن ع. شلبى و سونيا م.س. عزب و نهى أ. مجدى1و هانى م. توفيق2}

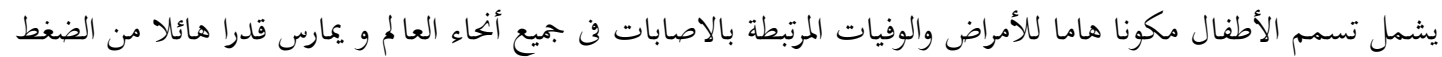

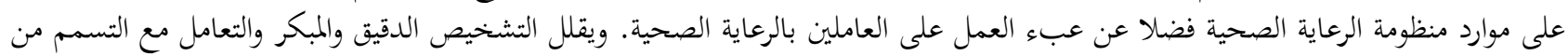

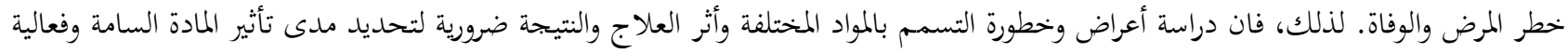

الهدف من الدراسة: هدف هذه الدراسة الى تحديد الوجهة الاكلينيكية والتعامل مع الأطفال المصابين بالتسمم الحاد والذين أدخلوا العلاج.

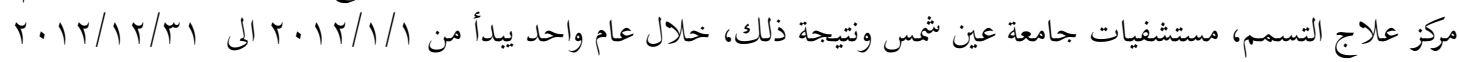

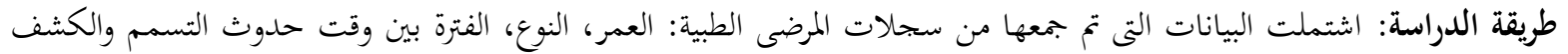

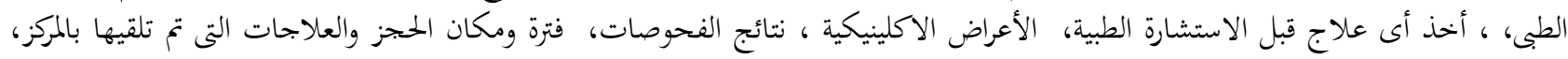

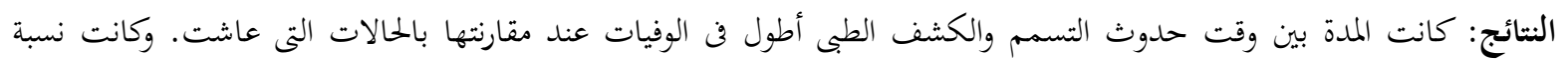

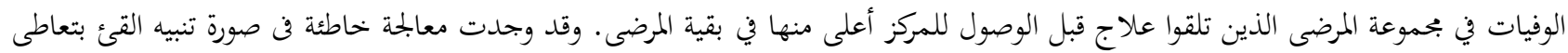

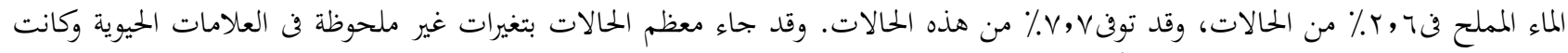

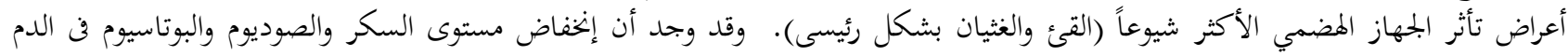

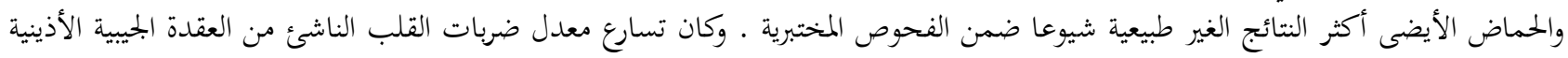
الشذوذ الأكثر شيوعا في رسم القلب الكهربائى الكئ.

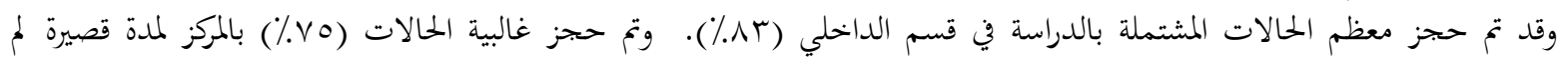

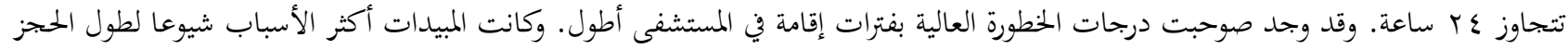
بالمستشفى (ك أيام)

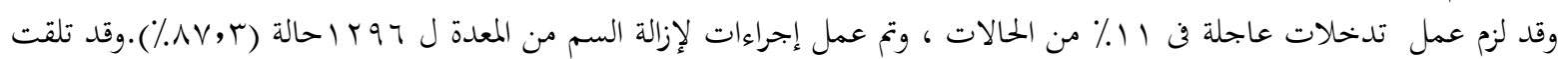

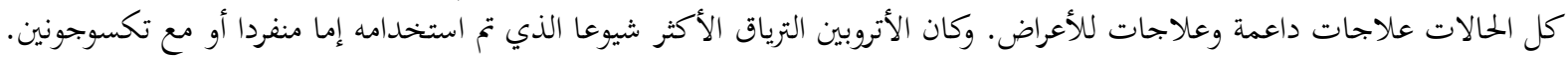

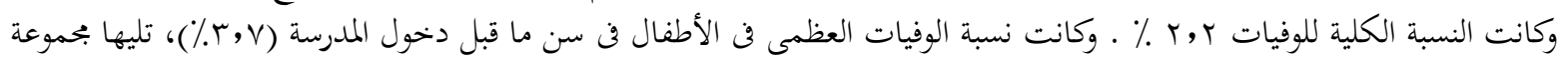

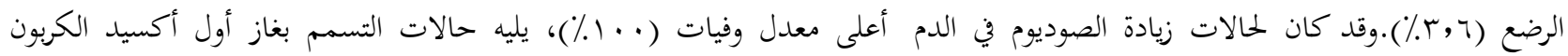

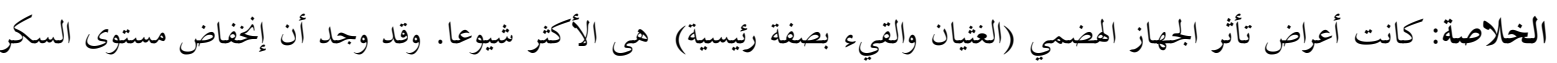

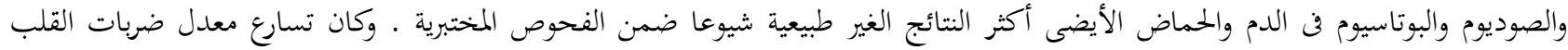

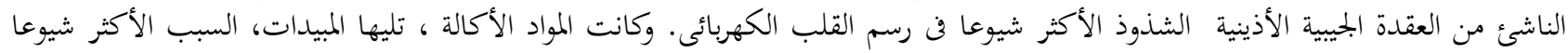

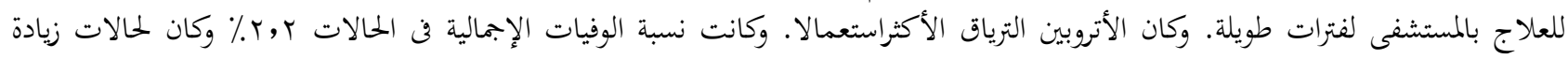

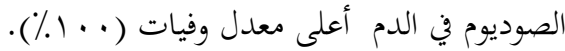

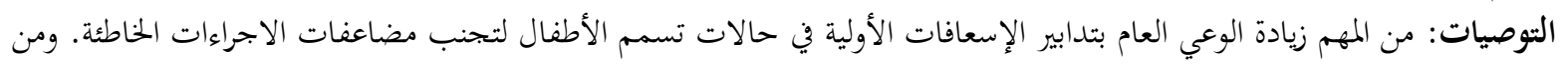

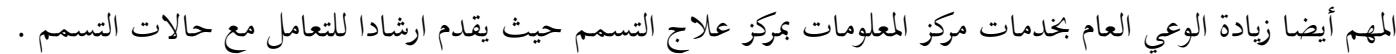

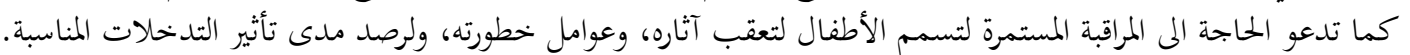

